\title{
Editorial: Flow and Transformation in Porous Media
}

\author{
Bjørnar Sandnes ${ }^{1 *}$, Daniel Koehn ${ }^{2}$, Renaud Toussaint ${ }^{3}$, Piotr Szymczak ${ }^{4}$ and \\ Einat Aharonov ${ }^{5}$
}

${ }^{1}$ Energy Safety Research Institute, College of Engineering, Swansea University, Swansea, UK, ${ }^{2}$ School of Geographical and Earth Sciences, University of Glasgow, Glasgow, UK, ${ }^{3}$ Institut de Physique du Globe de Strasbourg, Centre National de la Recherche Scientifique, University of Strasbourg/EOST, Strasbourg, France, ${ }^{4}$ Institute of Theoretical Physics, Faculty of Physics, University of Warsaw, Warsaw, Poland, ${ }^{5}$ Institute of Earth Sciences, Faculty of Mathematics and Sciences, Hebrew University of Jerusalem, Jerusalem, Israel

Keywords: porous media, granular materials, fracturing, pattern formation, capillary, friction, dissolution, two-phase flow

\section{The Editorial on the Research Topic}

\section{Flow and Transformation in Porous Media}

Fluid flow in transforming porous rocks, fracture networks, and granular media is subject to considerable current interdisciplinary research activity in Physics, Earth Sciences, and Engineering. Examples of natural and engineered processes include hydrocarbon recovery, carbon dioxide geo-sequestration, soil drying and wetting, pollution remediation, soil liquefaction, landslides, dynamics of wet or dry granular media, dynamics of faulting or friction, volcanic eruptions,

Edited by:

Alex Hansen,

Norwegian University of Science and

Technology, Norway

Reviewed by:

Daniel Bonamy,

French Alternative Energies and Atomic Energy Commission, France

*Correspondence: Bjørnar Sandnes b.sandnes@swansea.ac.uk

Specialty section

This article was submitted to Interdisciplinary Physics, a section of the journal Frontiers in Physics

Received: 24 August 2016 Accepted: 13 October 2016 Published: 28 October 2016

Citation: Sandnes B, Koehn D, Toussaint $R$ Szymczak P and Aharonov E (2016) Editorial: Flow and Transformation in

Porous Media. Front. Phys. 4:42. doi: 10.3389/fphy.2016.00042 gas venting in sediments, karst development and speleogenesis, ore deposit development, and radioactive waste disposal.

Hydrodynamic flow instabilities and pore scale disorder typically result in complex flow patterning. In transforming media, additional mechanisms come into play: compaction, de-compaction, erosion, segregation, and fracturing lead to changes in permeability over time. Dissolution, precipitation, and chemical reactions between solutes and solids may gradually alter the composition and structure of the solid matrix, either creating or destroying permeable paths for fluid flow. A complex, dynamic feedback thus arises where, on the one hand, the fluid flow affects the characteristics of the porous medium, and on the other hand the changing medium influences the fluid flow.

This Research Topic Ebook presents current research illustrating the depth and breadth of ongoing work in the field of flow and transformation in porous media through 15 papers by 72 authors from around the world. The body of work highlights the challenges posed by the vast range of length- and time-scales over which subsurface flow processes occur. Importantly, phenomena from each scale contribute to the larger-scale behavior. The flow of oil and gas in reservoirs, and the flow of groundwater on catchment scale is sensitively linked to pore scale processes and material heterogeneity down to the micrometer scale. The geological features of the same reservoirs and catchments evolved over millions of years, sometimes as a consequence of cracking and fracture growth occurring on the time scale of microseconds. The research presented by the authors of this Research Topic represents a step toward bridging the separation of scales as well as the separation of scientific disciplines so that a more unified picture of flow and transformation in porous media can start to emerge.

The first four papers in the Ebook focus on pore scale effects associated with capillary pressure, pore flow, and intergranular forces. Flovik et al. present a pore network model where a wettability 
altering agent is introduced to two-phase fluid flow. The model captures the intricate feedback between the changes in wettability and the evolving flow patterns. The authors are able to quantify the time dependent increase in oil fractional flow as trapped oil clusters are mobilized due to the change in wetting angle toward water-wetness. David et al. conduct a detailed experimental study of pore filling by imbibition of water into dry porous rocks. The authors use a combination of methods to characterize the motion of the capillary front. X-ray tomography imaging was performed in combination with ultrasound monitoring as a means of directly comparing the motion of the front with the acoustic signatures picked up by the ultrasound sensors.

Misztal et al. present a novel lattice Boltzmann simulation on unstructured grids of pollutant dispersion in the complex pore geometries of limestone samples discretized from X-ray tomography scans. The authors find that diffusive transport dominates over advective transport in pore spaces with high geometrical complexity. The results have implications for pollution remediation practices, suggesting that on/off cycles of flushing are more effective at removing pollutants compared to continuous flushing in the case of diffusion dominated systems. Marks et al. focus on the transmission of stress through graingrain contacts in compacting granular packings. The authors use Discrete Element Method (DEM) simulations to show that propagation of stress to confining walls leads to exponentially increasing boundary stresses as the compaction front thickens.

The next five papers focus on medium scale processes and pattern formation. Kelka et al. present a micro-scale model of the formation of bands in ore-bearing dolomites. The zebra pattern of white and dark stripes develops during mineral replacement and grain boundary migration that is influenced by second phase pinning. Grains in dark bands with a high concentration of impurities remain small whereas grains in white bands are almost impurity free and can develop large crystals. Eriksen et al. look at the formation of viscous fingering and fracturing patterns when two non-miscible fluids flow in a confined, but mobile, granular medium. The finger thickness and fractal dimension change significantly depending on whether deformable or undeformable boundary conditions are imposed, and whether or not the outer boundary is semi-permeable or free.

Turkaya et al. link observable granular deformation and fracture events to acoustic emissions in lab scale multiphase flow experiments associated with air injection. The authors are able to identify specific acoustic signatures associated with both the channel-creating fracturing events themselves, and aftershock emissions released during slow relaxation in compacted regions after the system has reached its final fractured state. Oppenheimer et al. present lab scale experiments on gas transport through high-viscosity, particle-rich suspensions serving as magma analogs. The authors demonstrate that an increased outgassing efficiency through fractures occurs when the crystal fraction is high, explaining why crystal-rich magmas are often observed to have depleted volatiles. Varas et al. present results from experiments of vertical gas venting through a submerged bed of grains where the granular material is fluidized within a parabolic-shaped process zone surrounding the rising gas column. The authors find a transition from intermittent to steady flow depending on gas flow rate and reservoir volume, and identify a new peculiar flow pattern where a gas "finger" extends vertically upwards to a critical height, above which the gas transport occurs through fractures in the granular packing.

The last six papers in this Research Topic deal with flow and transformation in reservoir scale geological systems. Keszthelyi et al. present a model to understand subsidence in the Ekofisk reservoir in the North Sea. The authors derive a micromechanical model combining microscopic processes of fracturing (pore failure) and creep (pressure solution) and upscale the model through fracture densities to understand reservoir scale behavior. Nermoen et al. present an experimental study of the effects of stress and temperature on chalk dissolution. In their long term experiments they flood core specimens of chalk with $\mathrm{MgCl}_{2}$ brine in a 3D deformation cell under stress and evaluate the mineralogical changes. Their experiments show an increase in exchange between $\mathrm{Mg}^{2+}$ and $\mathrm{Ca}^{2+}$ where the rock samples retain magnesium and lose calcium. This transformation is enhanced with an increase in stress and temperature.

Petrus and Szymczak present a numerical model of the development of solution pipes by $\mathrm{CO}_{2}$ saturated water flowing through limestone rocks and show how the pipes are influenced by layering in the soil. In their model the pipes develop through a reaction-infiltration instability that focuses flow due to a positive feedback between flow rate and dissolution driven porosity generation. The presence of low-porosity layers in the rock increases the competition between pipes so that flow focuses faster in single pipes and changes the shapes of the pipes locally, producing widening of pipes between the layers and narrowing of pipes in layers. Parez and Aharonov present simulations of sliding granular material to understand the run-off behavior of landslides. The authors show that the collapse of the landslides during their rapid travel results in a very low angle of repose, which leads to a long apparent runout even without any special low friction conditions at their base. Based on the simulations a scaling law is derived to connect landslide size with run-out and the authors show that this scaling is in accordance with data from natural examples.

Finally, there are two contributions on the evolution of fracture networks. Ghani et al. present a hydro-mechanical numerical model to simulate fluid driven fracturing in reservoirs with a special emphasis on layered systems. The authors present proxies to separate tectonically driven fracturing, with wellspaced and oriented networks of fractures, from fracturing that are driven mainly by fluid pressure gradients, which show breccia like patterns with variable fracture orientation. Sachau et al. present a numerical model of crustal scale hydraulic fractures and dynamic fluid movements. In their model, hydraulic fractures develop from an over-pressured source, travel upwards to release fluids and heal according to a viscous law. The authors explore the dynamics of their system and show that high healing rates lead to a very dynamic and pulse like fluid flow whereas low healing rates stabilize and result in constant flow.

The collective work presented by the authors demonstrate the growing interest in flow and transformation in porous media. 
The research field is progressing at an accelerating rate driven by the needs of industry and society, and by recent advances in experimental and theoretical tools. Researchers are now able to characterize pore geometries with unprecedented resolution using X-ray micro-tomography, produce micromodels of controllable porous media characteristics, and detect large scale events using remote sensing. Advances in high performance computing have made simulation of pore flow processes down to sub-pore resolution possible. Using a combination of experimental and theoretical methods, the researchers are starting to unravel the sensitive coupling between pore scale events and large-scale flow and transport processes. The sheer complexity of flow in transforming porous media manifested as evolution of preferential flow paths, pattern formation, intermittent dynamics, anomalous transport, and material fracturing poses enormous challenges, but problems once thought intractable are now starting to yield to scientific enquiry.

\section{AUTHOR CONTRIBUTIONS}

All authors listed, have made substantial, direct and intellectual contribution to the work, and approved it for publication.

\section{FUNDING}

This work has received funding from the European Union's Seventh Framework Programme for research, technological development, and demonstration under grant agreement no 316889. BS acknowledges support from EPSRC Grant No. EP/L013177/1 and Sêr Cymru National Research Network in Advanced Engineering and Materials Grant No. NRN141.

\section{ACKNOWLEDGMENTS}

We thank all authors, reviewers, and editors that have contributed to this Research Topic.

Conflict of Interest Statement: The authors declare that the research was conducted in the absence of any commercial or financial relationships that could be construed as a potential conflict of interest.

Copyright (c) 2016 Sandnes, Koehn, Toussaint, Szymczak and Aharonov. This is an open-access article distributed under the terms of the Creative Commons Attribution License (CC BY). The use, distribution or reproduction in other forums is permitted, provided the original author(s) or licensor are credited and that the original publication in this journal is cited, in accordance with accepted academic practice. No use, distribution or reproduction is permitted which does not comply with these terms. 